\title{
Recent advance in Immunotherapy of Acute Myeloid Leukemia
}

\author{
Nahla AM Hamed* \\ Hematology Department, Alexandria University, Egypt
}

Submission: August 08, 2018; Published: September 06, 2018

"Correspondence Address: Nahla AM Hamed, Professor of Hematology, Hematology Department, Faculty of Medicine, Alexandria University, Egypt, Email: drhamedn@hotmail.com

\begin{abstract}
Multiple malignancies showed incredible success with immunotherapy treatment. Immunotherapy for AML is now heavily researched being a highly promising strategy, particularly in transplant-ineligible patients and minimal residual disease states despite many challenges in treatment related to heterogeneous antigen expression, cytokine release syndrome and off target toxicities.

Abbreviations: AML: Acute Myeloid Leukemia; DCs: Dendritic Cells; NK: Natural Killer; PRAME: Preferentially Expressed Antigen in Melanoma; IR: Immune Response; CAR T cells: Chimeric-Antigen-Receptor T cells; LSA: Leukemia-Specific Antigens; LAA: Leukemia-Associated Antigens;
\end{abstract} PD-L1: Ligands for PD-1+ cells; HMA: Hypomethylating Agents;TCRtg: T-cell Receptor Gene-Transduced

\section{Introduction}

Myeloid cells (dendritic cells, macrophages, granulocytes, and platelets) play central role in immunity. They are critical in activating and sustaining adaptive and innate immunity. Immature myeloid cells, which resemble AML blasts in many respects, as well as mature myeloid cells can suppress immunity [1] and are key mediators of mature lymphocyte tolerance. Myeloid cells, particularly dendritic cells, localize to sites of B and $\mathrm{T}$ cell development where they tolerize lymphocyte precursors that recognize myeloid cell-associated antigens[1]. AML also evade the host immune system through downregulation of nonself-human leukocyte antigen, resistance to NK cells, decrease antigenicity, production of their own DCs leading to T-cell neutralization and release ligands to block T cell attacks[2].

Immunotherapy (defined as the generation of an efficacious anti-tumor IR) [3] has garnered much interest over the past several decades because it can destroy leukemic cells without the morbidity of a stem cell transplant[2]. Immunotherapeutic intervention aimed to increase response rates to chemotherapy, converting minimal residual disease, bridging to HSCT and treating refractory or relapsed patients [4].

\section{Patient Subsets most likely to Benefit from} Immunotherapy are

i. Older patient's ineligible for stem cell transplant.

ii. Relapsed/refractory AML patients after prior hematopoietic stem cell transplant. iii. Patients in complete recovery at high risk for relapse (minimal residual disease positive)[3].

Novel Immunotherapeutic approaches Currently Employed in AML clinical trials are
i. Immune checkpoint inhibitors,
ii. Therapeutic vaccination using DCs,
iii. Antibody-drug conjugates,
iv. T-cell recruiting antibody constructs,
v. TCR-transgenic T cells and
vi. Chimeric-antigen-receptor T cells (CAR T cells)[4], and
vii. Natural killer cell therapy[3].

\section{Monoclonal Antibodies}

Antigen-specific immunotherapies targeting various cell surface proteins on leukemic myeloblasts and leukemic stem cells include anti-CD47 (NCT02678338), anti-CD25 (NCT02588092), anti-CD56 (NCT02420873), ipilimumab (i.e., anti-CTLA4) (NCT01757639), and nivolumab (i.e., anti-PD1) (NCT02464657, NCT02397720), are in development in clinical trials and have not reported any in-depth results. Vadastuximab talirine (SGN$\mathrm{CD} 33 \mathrm{~A}$ ) is a novel antibody-drug conjugate. Vadastuximab is a CD33-directed antibody conjugated to pyrrolobenzodiazepine dimer. Vadastuximab has remarkable clinical activity with no off-target toxicity as a single agent and with HMAs in elderly 
patients[5]. So far, no conventional antibody has achieved approval for the treatment of AML[4].

\section{CART Therapy}

CARs are genetically engineered cell membrane-bound receptors that combine extracellular antibody binding and intracellular effector cell signaling, thereby enabling both MHC independent antigen binding and highly potent cytotoxic effector cell function[6]. Multiple targets include CD33, CD123, FRB, CLL1 or CLEC12A, FLT3, B7H6, NKG2D and Lewis y (LeY) are in development for directed CART therapy in AML. The biological heterogeneity of AML due to the different myeloid progenitors from which it arises is a challenge with the development of effective broad-spectrum CART for AML[3]. Several strategies are being explored to circumvent unwanted on target offleukemia toxicity resulting from persistence of current CART cell constructs beyond 4 years in the human body[5].

\section{Cancer Vaccine}

DC-based immunotherapy involves utilizing antigen-loaded DCs to induce a Th1 response which, in turn, generates cytotoxic $\mathrm{T}$ cells that target and lyse antigen-expressing tumor cells. In addition, peptide-based vaccine approaches have also been utilized to generate an antigen-specific IRs[3]. Vaccines are constructed from intact radiation inactivated AML cells, LAA or through LSA[2].LSA, are antigens specific to a tumor but may be expressed in only a minority of AML types (e.g., PML-RARA) while LAA, are antigens, associated with a tumor type but may be present on normal cell lines.AML cells express a multitude of LAA, including WT-1, PRTN3, PRAME and hTERT[3]. LSA are preferred targets to prevent inadvertent destruction of normal myeloid cell lines. LAA are more common but less specific, potentially inducing systemic toxicity to normal myeloid lines[2]. Cancer vaccine would be given in conjunction with standard chemotherapeutic regimens in the hope that they could prevent relapse through targeting chemo-refractory cells such as stem cells[2].

\section{Checkpoint Inhibitors}

Immune checkpoints are inherent methods to the immune system to prevent occurrence of autoimmunity. In normal circumstances, the checkpoint molecules prevent crossreactivity with self via T-cell receptor ligand binding and resultant inhibition of T-cell function. Tumors express PD-L1 thereby inducing T-cell dysfunction, or increase indoleamine 2,3-dioxygenase, an enzyme which catabolizes tryptophan leading to regulatory $\mathrm{T}$ cell formation. Long-term checkpoint molecules overexpression can reduce anti-tumor effect of therapy[2].

Monoclonal antibodies against checkpoint molecules block inhibitory signals like PD1 on T cells or PD-L1 on malignant cells, allowing for release of the "brakes" of anti-leukemic T cells. PD-1 seems to be upregulated on T-cells in relapsed and to some extent in newly diagnosed AML compared to healthy donors. The available data on PD-L1 expression in AML is equivocal. HMA may induce the expression of PD-1 and PD-L1 in T-cells and AML cells, respectively. This is supported by failure of checkpoint inhibitors monotherapy to produce a meaningful benefit for AML patients whereas promising clinical results were demonstrated with combination therapy with HMA[4]. PD-L1 upregulation on AML cells upon T-cell activation has been suggested as a potential resistance mechanism in an ex vivo system.

\section{T cell-Recruiting Antibody Constructs}

Several $\mathrm{T}$ cell-recruiting antibody constructs are under preclinical and early clinical development. The optimal antigen to target is still an open question. Novel class of molecules composed of the single-chain variable fragment of two antibodies of different specificity connected by a short peptide linker[5]. AMG 330 is a bispecific $\mathrm{T}$ cell engager (BiTE) construct which engages CD33 and CD3[7]. In contrast to the BiTE technology, dual-affinity re-targeting (DART) molecules are composed of heavy and light chain variable domains of two antigenbinding specificities $(A+B)$ on two independent polypeptide chains (VLA-VHB-VLB-VHA), which are stabilized through an additional C-terminal bridge. XmAb14045, developed by Xencor, is a structurally distinct anti-CD123 T cell-recruiting antibody construct in early clinical development[6]. Addition of a checkpoint inhibitor to T-cell recruiting antibodies might help to circumvent resistance[4].

\section{T-cell Receptor Gene-Transduced Lymphocytes}

This is based on an ex vivo generation of TCR-gene transduced T-cells using a retroviral vector. They target a degraded, processed and presented antigen in the context of HLA molecules on the surface of the malignant cell. WT1 is the dominant antigen for TCRtg based immunotherapeutic strategies. Very limited data has been published on adoptive transfer of TCRtg cells in AML patients[4].

\section{Natural killer Cell Therapy}

Based on drastic alteration in NK activity in leukemic conditions, multiple immunotherapeutic strategies directed at enhancing effective NK cell function are being prospectively evaluated as AML therapy. Approaches to enhance the clinical efficacy of allogeneic NK cells include: using NK cells in conjunction with allogeneic stem cell transplant; improving delivery of a diverse NK cell repertoire and/or functional subsets; activation or priming of NK cells to induce greater anticancer activity; and generation of autologous NK cells with CART constructs[3].

\section{Obstacles to Successful Implementation of Immunotherapy in AML \\ a. Identifying suitable intracellular and surface target antigens[4].In AML, it is more difficult to choose an}


appropriate target antigen due to [6]heterogeneous tumor antigen expression in diverse AML subtypes [3] and a more ubiquitous expression pattern overlapping with healthy hematopoiesis[6].

b. Myeloid-associated lineage antigens like CD33 (expressed on $>30 \%$ of healthy bone marrow cells), [6] CD123, CLL-1 and FLT3 are also expressed within the healthy myeloid compartment [4]so Off-target toxicities to normal myeloid progenitor and hematopoietic stem cells may occur. It is expected that targeting AML-associated antigens will result in prolonged drug-induced cytopenias [6]as well as life-threatening cytokine release syndrome and neurological events[4]. This will require the adjustment of current protocols applied in ALL to the different setting in AML. Various potential target antigens are studied for each of the immunotherapeutic strategies[6].

c. Immune cell exhaustion.

d. Life-threatening complications of potent immune activation[3].

e. Low endogenous immune responses in AML[4].

f. Intrinsic resistance mechanisms of the leukemic blasts against immune response[6]. g. High tumor burden and clonal evolution of tumor cells over time[6].

\section{Conclusion}

Immunotherapy in AML will induce the best treatment responses for minimal residual disease eradication after induction chemotherapy. The optimal time point for immunotherapeutic intervention is poorly defined.

\section{References}

1. Geiger TL, Rubnitz JE (2015) New approaches for the immunotherapy of acute myeloid leukemia. Discov Med 19(105): 275-284.

2. Nagler E, Xavier MF, Frey N (2017) Updates in immunotherapy for acute myeloid leukemia. Transl Cancer Res 6(1): 86-92.

3. Przespolewski A, Szeles A, Wang ES (2018) Advances in immunotherapy for acute myeloid leukemia. Future Oncol 14(10): 963-978.

4. Subklewe M (2018) Current status of immunotherapy in acute myeloid leukemia. Educational Updates in Hematology Book 2(S2): 15-17.

5. Saygin C, Carraway HE (2017) Emerging therapies for acute myeloid leukemia. Journal of Hematology \& Oncology 10(1): 93.

6. Lichtenegger FS, Krupka C, Haubner S, Köhnke T, Subklewe M (2017) Recent developments in immunotherapy of acute myeloid leukemia. J Hematol Oncol 10(1): 142.

7. Tefferi A, Estey EH Acute myeloid leukemia: 2016 Update on riskstratification and management. Am J Hematol 91(8): 824-846.

\section{Your next submission with Juniper Publishers will reach you the below assets}

- Quality Editorial service

- Swift Peer Review

- Reprints availability

- E-prints Service

- Manuscript Podcast for convenient understanding

- Global attainment for your research

- Manuscript accessibility in different formats ( Pdf, E-pub, Full Text, Audio)

- Unceasing customer service

Track the below URL for one-step submission https://juniperpublishers.com/online-submission.php 